\title{
Impact of Indocyanine Green for Sentinel Lymph Node Mapping in Early Stage Endometrial and Cervical Cancer: Comparison with Conventional Radiotracer ${ }^{99 \mathrm{~m}} \mathrm{Tc}$ and/or Blue Dye
}

\author{
Alessandro Buda, MD ${ }^{1,2}$ (D) Cinzia Crivellaro, $\mathrm{MD}^{2,3}$, Federica Elisei, $\mathrm{MD}^{3}$, Giampaolo Di Martino, MD ${ }^{1,2}$, \\ Luca Guerra, $\mathrm{MD}^{3}$, Elena De Ponti, $\mathrm{PhD}^{4}$, Marco Cuzzocrea, $\mathrm{MD}^{2}$, Daniela Giuliani, $\mathrm{MD}^{1,2}$, Federica Sina, $\mathrm{MD}^{1,2}$, \\ Sonia Magni, $\mathrm{MD}^{1,2}$, Claudio Landoni, $\mathrm{MD}^{2,3,5}$, and Rodolfo Milani, $\mathrm{MD}^{1,2}$ \\ ${ }^{1}$ Gynecologic Oncology Surgery Unit, Department of Obstetrics and Gynecology, San Gerardo Hospital, Monza, Italy; \\ ${ }^{2}$ University of Milano-Bicocca, Monza, Italy; ${ }^{3}$ Department of Nuclear Medicine, San Gerardo Hospital, Monza, Italy; \\ ${ }^{4}$ Department of Medical Physics, San Gerardo Hospital, Monza, Italy; ${ }^{5}$ Technomed Foundation, University of Milano- \\ Bicocca, Monza, Italy
}

\begin{abstract}
Purpose. To compare the detection rate (DR) and bilateral optimal mapping (OM) of sentinel lymph nodes (SLNs) in women with endometrial and cervical cancer using indocyanine green (ICG) versus the standard technetium-99m radiocolloid $\left({ }^{99 \mathrm{~m}} \mathrm{Tc}\right)$ radiotracer plus methylene or isosulfan blue, or blue dye alone.

Methods. From October 2010 to May 2015, 163 women with stage I endometrial or cervical cancer (118 endometrial and 45 cervical cancer) underwent SLN mapping with ${ }^{99 \mathrm{~m}}$ Tc with blue dye, blue dye alone, or ICG. DR and bilateral OM of ICG were compared respectively with the results obtained using the standard ${ }^{99 \mathrm{~m}} \mathrm{Tc}$ radiotracer with blue dye, or blue dye alone.

Results. SLN mapping with ${ }^{99 \mathrm{~m}}$ Tc radiotracer with blue dye was performed on 77 of 163 women, 38 with blue dye only and 48 with ICG. The overall DR of SLN mapping was 97,89 , and $100 \%$ for ${ }^{99 \mathrm{~m}} \mathrm{Tc}$ with blue dye, blue dye alone, and ICG, respectively. The bilateral OM rate for ICG was $85 \%$-significantly higher than the $58 \%$ obtained with ${ }^{99 \mathrm{~m}} \mathrm{Tc}$ with blue dye $(p=0.003)$ and the $54 \%$ for blue dye $(p=0.001)$. Thirty-one women $(19 \%)$ had positive SLNs. Sensitivity and negative predictive value of SLN were $100 \%$ for all techniques.
\end{abstract}

(C) Society of Surgical Oncology 2015

First Received: 20 August 2015;

Published Online: 29 December 2015

A. Buda, MD

e-mail: ginoncmonza@gmail.com
Conclusions. SLNs mapping using ICG demonstrated higher DR compared to other modalities. In addition, ICG was significantly superior to ${ }^{99 \mathrm{~m}} \mathrm{Tc}$ with blue dye in terms of bilateral OM in women with early stage endometrial and cervical cancer. The higher number of bilateral OM may consequently reduce the overall number of complete lymphadenectomies, reducing the duration and additional costs of surgical treatment.

Sentinel lymph node (SLN) mapping represents the standard of care for treatment of breast and melanoma tumors. In gynecologic cancer, it is also widely accepted for vulvar cancer, following the publication of the GOG173 and GROINSS V-1 studies. ${ }^{1,2}$ Over the last few decades, SLN mapping has received increasingly more attention in the treatment of cervical and endometrial cancers, the objective being to minimize treatment-related morbidity and to prolong the benefits of surgical staging. Regarding the approach, the cervix represents the most accessible, easiest, fastest, and most reproducible site for dye and/or radiotracer injection. ${ }^{3-5}$ Since the preliminary experience of Burke et al. was published, the most widely investigated tracers for SLN mapping are technetium-99m radiocolloid $\left({ }^{99 \mathrm{~m}} \mathrm{Tc}\right.$ ) and blue dye (methylene or isosulfan blue). ${ }^{6}$ The detection rate (DR) of SLN is reported to be close to $80 \%$ in recent studies, while optimal bilateral mapping in minimally invasive surgery ranges from 50 to $61 \%$, even in reference centers. ${ }^{7-10}$

Therefore, to enhance the benefits of SLN mapping aimed at avoiding unnecessary pelvic and aortic lymphadenectomy, thereby reducing surgical morbidity, 
fluorescent indocyanine green (ICG) dye has been used and tested by several groups in SLN mapping in various solid tumors. ${ }^{11}$ Near-infrared (NIR) fluorescent dye with ICG has a higher signal-to-background ratio, is cheaper, has fewer adverse effects and less toxicity, and has infrequent allergic reactions. $^{12}$

The purpose of this study was to evaluate the DR of SLNs in endometrial and cervical malignancies using ICG and NIR fluorescent imaging compared to ${ }^{99 \mathrm{~m}} \mathrm{Tc}$ with blue dye and blue dye alone. The study was performed at the San Gerardo Hospital in Monza, Italy. Because side-specific lymphadenectomy is required in cases where only unilateral SLN mapping is achieved, optimal mapping, defined as the identification of at least 1 SLN for each hemipelvis, was also evaluated and compared in these groups of patients.

\section{MATERIALS AND METHODS}

The retrospective study lasted from October 2010 to May 2015. All consecutive cases of preoperative stage I endometrial cancer and stage I (1A2-1B1) cervical cancer were identified and included in this study. The approval of our institutional review board was obtained for this study, and all the participants provided written informed consent. All patients underwent total simple or radical hysterectomy with bilateral salpingo-oophorectomy and bilateral pelvic lymphadenectomy that included SLN mapping. Frozen sectioning was not performed. All the SLNs were sent separately for examination. Aortic lymphadenectomy was performed on the cervical cancer patients with grossly enlarged pelvic nodes, or in endometrial cancer patients with a positive preoperative positron emission tomography/computed tomography (PET/CT) scan and in the absence of SLN mapping or unilateral mapping. We began SLN mapping in 2010. After the completion of the learning curve and initial experience, we moved in the direction of an injection of blue dye alone into the cervix before ICG injection, which started in 2014.

\section{SLN Mapping Procedures}

From October 2010 until February 2014, the SLN mapping procedure was performed as previously described by using ${ }^{99 \mathrm{~m}}$ Tc plus blue dye or blue dye alone. ${ }^{13}$ The injection technique of the tracers was the same for both cervical and endometrial cancer. Specifically, a total of $4 \mathrm{~mL}$ of blue dye ( $2 \mathrm{~mL}$ per injection for each side) was injected at the 3 and 9 o'clock positions. The 12 o'clock injection was eliminated in order to avoid the blue staining the vesicocervicovaginal space, which would make bladder dissection more difficult.

In February 2014, fluorescent detection with ICG (Pulsion Medical Systems SE, Feldkirchen, Germany) was introduced in our department using Storz SPIES Full HD D-Light P ICG technology (Karl Storz, Mittelstrasse, Tuttlingen, Germany) for both minimally invasive and open procedures (Vitom II exoscope). The ICG concentration used was $1.25 \mathrm{mg} / \mathrm{mL}$. For each patient, a $25 \mathrm{mg}$ vial with ICG powder was diluted in $20 \mathrm{~mL}$ of aqueous sterile water. A total of $4-5 \mathrm{~mL}$ of this ICG solution were injected into the cervix alone, divided into the 3- and 9o'clock positions. One milliliter of ICG solution was injected with penetration to a depth of $1 \mathrm{~cm}$ into the stroma, and $1 \mathrm{~mL}$ was injected into the submucosal layer on the right and the left of the cervix, usually after initial laparoscopic exploration to evaluate the feasibility of the surgical procedure. For open surgical cases performed with the Vitom II exoscope, the cervical injection was performed once the laparotomy was completed. During the procedure, the lights in the operating room were switched off so the surgeons could view the HD Vitom II images displayed on a 26-inch flat-screen monitor installed at a convenient viewing distance and angle.

\section{Pathologic Evaluation}

The disease of all women was staged after definitive histologic results according to the 2009 International Federation of Gynecology and Obstetrics classification. An expert gynecologic oncology pathologist, highly skilled in the analysis of SLNs, examined all surgical samples and SLNs. All lymph nodes (LNs) were handled in a standardized manner. ${ }^{14}$ LNs with macroscopic metastases were sectioned, and SLNs that appeared normal were cut perpendicular to the long axis. Two adjacent $5 \mu \mathrm{m}$ sections were cut at each of 2 levels $50 \mu \mathrm{m}$ apart from each block lacking metastatic carcinoma, detected by means of a section routinely stained with hematoxylin and eosin (H\&E). At each level, one slide was stained with $H \& E$ and the other with immunohistochemistry using AE1/AE3, an anti-cytokeratin antibody (Dako, Glostrup, Denmark), as well as one other negative control slide, for a total of five slides per block. All other non-SLNs were examined only by routine $\mathrm{H} \& \mathrm{E}$. Micrometastasis was defined as a metastatic deposit within the LNs ranging from $0.2 \mathrm{~mm}$ to no more than $2 \mathrm{~mm}$ in size. Isolated tumor cells were defined as single tumor cells or as clusters of malignant epithelial cells less than $0.2 \mathrm{~mm}$ in size.

\section{Statistical Analysis}

Absolute and percentage frequencies were used to describe categorical items, while median values, standard deviation, and range were assessed for continuous characteristics. The rank sum test and Fisher's exact test were used to analyze the differences between the ICG group, 
${ }^{99 \mathrm{~m}} \mathrm{Tc}$ radiotracer with blue dye, or blue dye alone. A level of $p<0.05$ was adopted for significance. Stata 9.0 software (StataCorp, College Station, TX) was used to perform the statistical analysis.

\section{RESULTS}

\section{Patient Population}

One hundred sixty-three women were evaluated, comprising 118 with early stage endometrial cancer and 45 with 1A2-1B1 cervical cancer. The general characteristics of the study population are listed in Table 1 .

Median body mass index was $34 \mathrm{~kg} / \mathrm{m}^{2}$ (range 18$50 \mathrm{~kg} / \mathrm{m}^{2}$ ) and $26 \mathrm{~kg} / \mathrm{m}^{2}$ (range $16-35 \mathrm{~kg} / \mathrm{m}^{2}$ ) for women with endometrial and cervical cancer, respectively. In $70 \%$ of the cases (115 of 163), the surgical procedure was laparoscopy.

The total number of dissected LNs was 2727 overall; $384(14 \%)$ were SLNs. The median number of SLNs per hemipelvis was 2 (SD 0-9). Systematic pelvic lymphadenectomy was performed on all patients, and aortic lymphadenectomy was performed in 19 women (17 patients with endometrial cancer and two patients with cervical cancer). The median number of pelvic LNs removed was 29 (range 4-54) and 25 (range 6-58) in women with endometrial and cervical cancer, respectively. In the women with endometrial cancer, aortic lymphadenectomy was performed in $14 \%$ of cases (17 of 118). The median number of aortic nodes was 15 (range 2-29). Globally, SLNs were most frequently located in the external iliac region (71\%), internal iliac (2\%), obturator fossa $(10 \%)$, common iliac (12\%) and para-aortic (2\%), sacral $(2 \%)$, and parametrial $(1 \%)$. The number and anatomic locations of SLNs are shown in Fig. 1.

\section{Comparison of SLN Mapping Modalities}

The overall DR of SLN mapping was 97, 89, and $100 \%$ for ${ }^{99 \mathrm{~m}} \mathrm{Tc}$ with blue dye, blue dye alone, and ICG, respectively (Table 2).

The SLN mapping failed in six cases (3.8\%): 2 cases of ${ }^{99 \mathrm{~m}} \mathrm{Tc}$ with blue dye and 4 cases of blue dye only. The bilateral DR was $64 \%$ (105 of 163); bilateral mapping using ${ }^{99 \mathrm{~m}} \mathrm{Tc}$ with blue dye, blue dye alone, and ICG were $58 \%$ (45 of 77), $50 \%$ (19 of 38), and $85 \%$ (41 of 48), respectively. Successful bilateral mapping was significantly higher with ICG compared to either ${ }^{99 \mathrm{~m}} \mathrm{Tc}$ with blue dye $(p=0.003)$ or blue dye alone $(p=0.001)$. Surgical and LN characteristics are shown in Table 2.

Interestingly, in cervical cancer patients, the bilateral mapping was $23(82 \%)$ of $28,5(62 \%)$ of 8 , and 9
(100\%) of 9 for ${ }^{99 \mathrm{~m}} \mathrm{Tc}$ with blue dye, blue dye alone, and ICG, respectively. In the endometrial cancer cases, the ICG DR was $100 \%$ and demonstrated the highest rate of bilateral mapping compared to other methods. SLN mapping differences according to tumor are shown in Table 3.

\section{Histopathologic Analysis}

LN metastases were discovered in $31(19 \%)$ of 163 women. Three women $(10 \%)$ had SLN metastases that were detected only through additional sectioning upon ultrastaging. Twenty-three percent of SLN positivity included $19 \%$ of micrometastasis and $4 \%$ of isolated tumor cells. In patients with no SLN mapping, no positive LNs were discovered during final routine pathology. Furthermore, in cases of unilateral SLN detection, only 4 $(8 \%)$ of 50 patients presented nodal involvement on the other side, with a total of six LNs involved (one micrometastasis, five macrometastasis). In patients with no SLN mapping, no positive LNs were discovered during final routine pathology. Moreover, in cases of unilateral SLN detection, only $4(8 \%)$ of 50 of the patients presented nodal involvement on the other side, with a total of $6 \mathrm{LNs}$ involved (one micrometastasis, five macrometastasis). Positive SLN characteristics are shown in Table 2.

Patients with positive LNs underwent further therapy according to their risk factors at final histology. Cervical cancer patients $(n=10)$ received adjuvant radiochemotherapy, whereas endometrial cancer patients $(n=21) \quad$ underwent chemotherapy $\quad(n=10)$ or radiochemotherapy $(n=9)$. Two women did not receive adjuvant therapy because an impaired general condition and comorbidity contraindicated further therapy. Considering all modalities (ICG, blue dye, and ${ }^{99 \mathrm{~m}} \mathrm{Tc}$ with blue dye), the false-negative rate per patients was 0 because SLN negativity with non-SLNs positive for metastasis was not found. The negative predictive value was $100 \%$.

\section{DISCUSSION}

$\mathrm{LN}$ involvement still remains the most important prognostic factor for women with endometrial and cervical malignancy. However, because early stage disease has a low risk of LN metastasis, in the last few decades, SLN mapping has received increased attention. It is a technique that could potentially result in reducing the morbidity associated with ineffective complete lymphadenectomy. ${ }^{15}$

Since Ballester et al. published their preliminary SLN mapping experience with cervical injection for women with endometrial cancer, a great amount of valuable literature has been produced. ${ }^{8}$ There are data supporting its usefulness in detecting micrometastases, thanks to the ultrastaging 
TABLE 1 Patient's characteristics $(N=163)$

\begin{tabular}{|c|c|c|c|c|}
\hline Characteristic & ${ }^{99 \mathrm{~m}} \mathrm{Tc}+$ blue dye $(N=77)$ & Blue alone $(N=38)$ & $\mathrm{ICG}(N=48)$ & $p$ value \\
\hline \multicolumn{4}{|l|}{ Age (years) } & a versus b: $0.900^{\S}$ \\
\hline Median \pm SD (range) & $61 \pm 13.4(26-86)$ & $62 \pm 13.2(29-86)$ & $59 \pm 14.5(29-86)$ & $\begin{array}{l}\text { a versus c: } 0.903^{\S} \\
\text { b versus c: } 0.865^{\S}\end{array}$ \\
\hline \multicolumn{4}{|l|}{ BMI $\left(\mathrm{kg} / \mathrm{m}^{2}\right)$} & a versus b: $0.026^{\S}$ \\
\hline Median \pm SD (range) & $23 \pm 4.9(18-50)$ & $26 \pm 5.3(18-40)$ & $25 \pm 6.6(15-50)$ & $\begin{array}{l}\text { a versus c: } 0.032^{\S} \\
\text { b versus c: } 0.746^{\S}\end{array}$ \\
\hline \multicolumn{4}{|l|}{ Type of tumor } & a versus b: $0.071^{*}$ \\
\hline Cervical & $28(36.4 \%)$ & $8(21.1 \%)$ & $9(18.8 \%)$ & a versus c: $0.027 *$ \\
\hline Endometrial & $49(63.6 \%)$ & $30(78.9 \%)$ & $39(81.2 \%)$ & b versus c: $0.500^{*}$ \\
\hline \multicolumn{5}{|l|}{ pT stage (final pathology) } \\
\hline \multicolumn{4}{|l|}{ Cervical } & a versus b: $0.163^{*}$ \\
\hline IA2 & $3(10.7 \%)$ & $2(28.6 \%)$ & $2(22.2 \%)$ & a versus c: $0.765^{*}$ \\
\hline IB 1 & $23(82.1 \%)$ & $4(57.1 \%)$ & $7(77.8 \%)$ & $\mathrm{b}$ versus c: $0.758^{*}$ \\
\hline IIA & $2(7.1 \%)$ & - & - & \\
\hline IIB & - & $1^{\mathrm{a}}(14.3 \%)$ & - & \\
\hline \multicolumn{4}{|l|}{ Endometrial } & a versus b: $0.581 *$ \\
\hline EIN & - & - & $2(5.1 \%)$ & a versus c: $0.070^{*}$ \\
\hline IA & $23(46.9 \%)$ & $17(56.7 \%)$ & $27(69.2 \%)$ & b versus c: $0.141^{*}$ \\
\hline IB & $10(20.4 \%)$ & $8(26.7 \%)$ & $3(7.7 \%)$ & \\
\hline II & $4(8.2 \%)$ & - & $1(2.6 \%)$ & \\
\hline IIIA & - & - & $1(2.6 \%)$ & \\
\hline IIIC1 & $10(20.4 \%)$ & $5(16.7 \%)$ & $4(10.3 \%)$ & \\
\hline IIIC2 & $1(2.0 \%)$ & - & - & \\
\hline IV & $1(2.0 \%)$ & - & $1(2.6 \%)$ & \\
\hline
\end{tabular}

Histology (final pathology)

Cervical

\section{Squamous}

Adenocarcinoma

Endometrial

EIN

Endometrioid

Serous papillary

Other

Grade

$\begin{array}{ll}\text { G1 } & 19(25.7 \%) \\ \text { G2 } & 31(41.9 \%) \\ \text { G3 } & 24(32.4 \%) \\ \text { NA } & 3 \# \\ \text { LSI } & \\ \text { Yes } & 31(40.3 \%) \\ \text { No } & 46(59.7 \%)\end{array}$

$18(64.3 \%)$

$10(35.7 \%)$

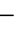

$41(83.7 \%)$

$2(4.1 \%)$

$6(12.2 \%)$

$46(59.7 \%)$
$7(87.5 \%)$

$1(12.5 \%)$

$-$

$27(90.0 \%)$

$1(3.3 \%)$

$2(6.7 \%)$

$10(27.0 \%)$
$19(51.4 \%)$
$8(21.6 \%)$
$1^{\wedge}$

$14(36.8 \%)$

$24(63.2 \%)$
$6(66.7 \%)$

$3(33.3 \%)$

$2(5.1 \%)$

$33(84.6 \%)$

$3(7.7 \%)$

$1(2.6 \%)$

$15(31.2 \%)$

$17(35.5 \%)$

$16(33.3 \%)$

$-$

$10(20.8 \%)$

$38(79.2 \%)$ a versus b: $0.210^{*}$

a versus c: $0.614^{*}$

b versus c: $0.335^{*}$

a versus b: $0.867 *$ a versus c: $0.138^{*}$ b versus c: $0.573 *$

a versus b: $0.487^{*}$ a versus c: $0.733^{*}$ b versus c: $0.327 *$

a versus b: $0.442 *$ a versus c: $0.019^{*}$ b versus c: 0.081 *

SD standard deviation, ${ }^{99 m} T c$ technetium-99 radiocolloid, ICG indocyanine green, NA not available, LVSI lymphovascular space involvement, $B M I$ body mass index

${ }^{a}$ Woman with clinical 1B1 cervical cancer demonstrated foci of tumor that involved both parametrial tissues at final pathology (stage pT IIB disease)

* Fisher's exact test; ${ }^{\S}$ Rank sum test; \# EIN endometrial intraepithelial neoplasia; ^ $C I S$ cervical intraepithelial in situ 
FIG. 1 SLN anatomic distribution and metastasis location in the study population. In our population, 384 SLNs were obtained by all modalities. Number of SLNs per site indicated by circles; number of positive SLNs at histology indicated by triangles. *Four SLNs were in parametrial site. SLN sentinel lymph node

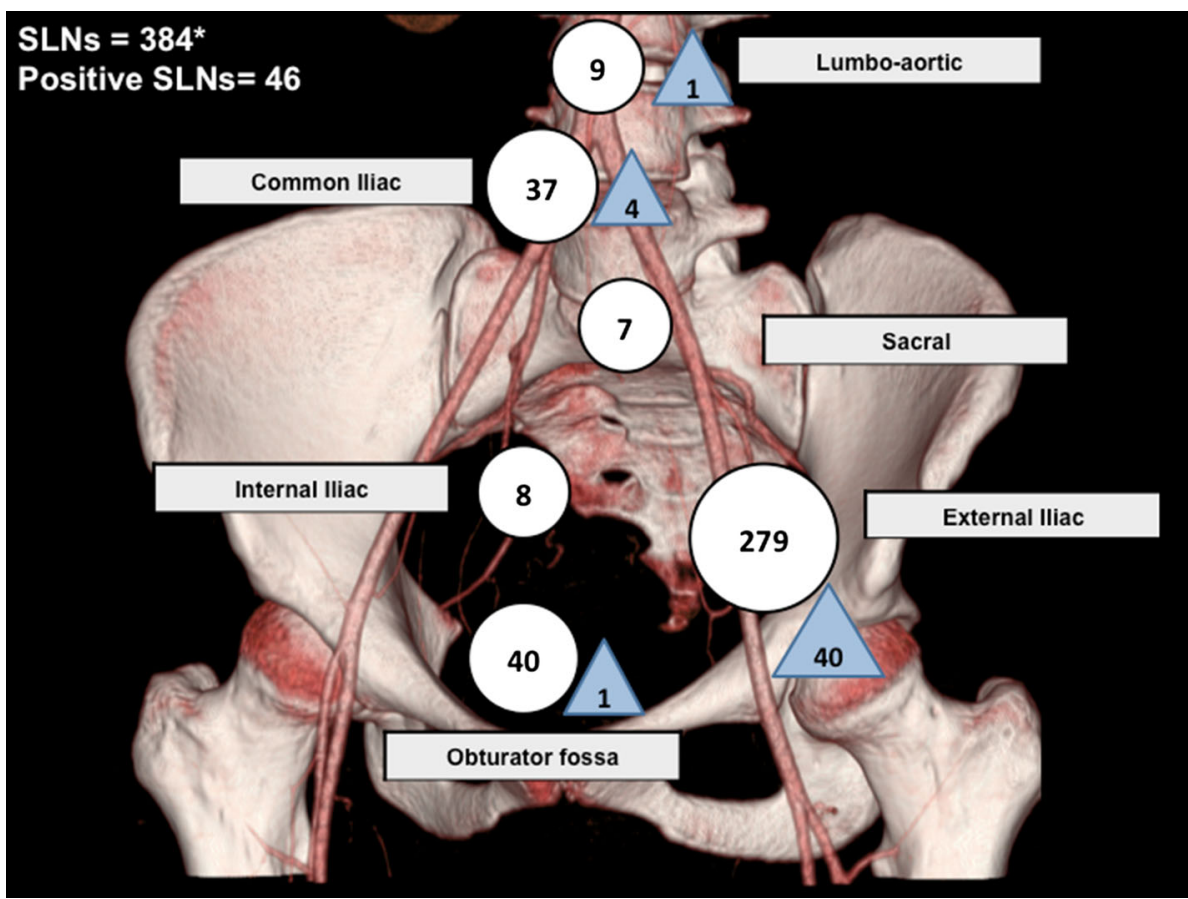

technique, which allows for a lower complication rate than radical pelvic and/or aortic lymphadenectomy. ${ }^{16,17}$

In 2015, SLN mapping was incorporated into the National Comprehensive Cancer Network guidelines for both cervical and endometrial cancer in highly specialized centers with extensive experience in SLN mapping and included applying a SLN algorithm to reduce the falsenegative rate. ${ }^{18-20}$ Moreover, in cervical cancer patients, a large retrospective multicenter study showed that the early detection of micrometastasis in patients with cervical cancer has the same impact on survival as in cases of macrometastasis. ${ }^{21}$

The standard for the most effective SLN mapping in both early stage cervical and endometrial cancers is a dual technique including the combination of ${ }^{99 \mathrm{~m}} \mathrm{Tc}$ and blue dye. In the literature, the SLN DR with both blue dye and ${ }^{99 \mathrm{~m}} \mathrm{Tc}$ ranges from 80 to $100 \%{ }^{7}$ However, the use of a radiolabeled tracer is more demanding for hospitals because it presents many logistic challenges and costs that limit their use worldwide, particularly for countries with limited or no access to radioisotopes.

More recently, the clinical effectiveness of NIR fluorescence using ICG has been evaluated for SLN mapping in gynecologic malignancies, particularly in a minimally invasive, traditionally laparoscopic approach or using a robotic platform. ${ }^{10,22-24}$ The U.S. Food and Drug Administration has approved ICG for use in humans since 1959 for cardiac and liver functions, but for tissue ICG, its use is off label. Moreover, ICG is widely used particularly for chorioangiography and as a tool to delineate the extrahepatic biliary tree for NIR fluorescence cholangiography.
To date, and including our study, about 400 cases have been published, and globally, the DR and bilateral detection seem to be superior to ${ }^{99 \mathrm{~m}} \mathrm{Tc}$ with or without blue dye. We started SLN mapping in October 2010 in our department, and more recently we have introduced real-time fluorescent SLN detection with ICG. ${ }^{13}$ Many recent studies have demonstrated that NIR fluorescence seems to be safe and feasible, and also allows for real-time observation of lymphatic channels with high DRs and low false-negative rates.

An extensive study using ICG in gynecologic cancers was recently published by Jewell et al. ${ }^{10}$ The optimal bilateral mapping of ICG alone was $79 \%$ (156 of 197) and for ICG and blue dye was $77 \%$ (23 of 30). The authors concluded that the intracervical injection of ICG has a high bilateral DR and appears to offer an advantage over using blue dye alone, so that the combined use of ICG and blue dye appears unnecessary. To our knowledge, this is the first European experience of SLN mapping with fluorescent ICG, using the Storz ICG SPIES system for the laparoscopic platform and traditional open surgery using the Vitom II system.

The intracervical injection of ICG improved the optimal bilateral mapping (58\% up to $85 \%$ ), with a consequent important impact on the management of our cases. In our series of 163 consecutive cases, SLNs mapping using ICG demonstrated the highest DR compared to other modalities $(100 \%)$. In addition, florescent mapping with ICG was significantly superior to ${ }^{99 \mathrm{~m}} \mathrm{Tc}$ with blue dye or blue dye alone in terms of bilateral mapping in early stage endometrial and cervical cancer (85\% ICG vs. 58 and 
TABLE 2 Surgical procedure and pathological characteristics of LNs and SLNs $(N=163)$

\begin{tabular}{|c|c|c|c|c|}
\hline Characteristic & ${ }^{99 \mathrm{~m}} \mathrm{Tc}+$ blue dye $(N=77)$ & Blue alone $(N=38)$ & ICG $(N=48)$ & $p$ value \\
\hline Surgical approach & & & & a versus b: $0.338^{*}$ \\
\hline MIS & $46(59.7 \%)$ & $25(65.8 \%)$ & $44(91.7 \%)$ & a versus $\mathrm{c}:<0.0001 *$ \\
\hline Open & $31(40.3 \%)$ & $13(34.2 \%)$ & $4(8.3 \%)$ & b versus c: $0.003^{*}$ \\
\hline Type of surgery & & & & a versus b: $0.111^{*}$ \\
\hline $\mathrm{SH}+\mathrm{BSO}+\mathrm{PLND}$ & $37(48.1 \%)$ & $27(71.1 \%)$ & $31(64.6 \%)$ & a versus c: $0.166^{*}$ \\
\hline $\mathrm{SH}+\mathrm{BSO}+\mathrm{PLND}+$ omentectomy & $2(2.6 \%)$ & $1(2.6 \%)$ & $3(6.3 \%)$ & b versus c: $0.698 *$ \\
\hline $\mathrm{SH}+\mathrm{BSO}+\mathrm{PLND}+\mathrm{PALND}$ & $10(13.0 \%)$ & $2(5.3 \%)$ & $5(10.4 \%)$ & \\
\hline $\mathrm{RH}+\mathrm{PLND}$ & $27(35.1 \%)$ & $7(18.4 \%)$ & $9(18.8 \%)$ & \\
\hline $\mathrm{RH}+\mathrm{PLND}+\mathrm{PALND}$ & $1(1.3 \%)$ & $1(2.6 \%)$ & - & \\
\hline \multicolumn{5}{|l|}{ LN surgical staging } \\
\hline Pelvic LNs, median (range) & $25(6-54)$ & $22(4-58)$ & $23(7-33)$ & \\
\hline Aortic LNs, median (range) & $15(2-29)$ & $10(2-18)$ & $8(4-17)$ & \\
\hline SLN per patients, median (range) & $2(0-9)$ & $2(0-4)$ & $2(0-6)$ & $\begin{array}{l}\text { a versus b: } 0.0001^{\S} \\
\text { a versus c: } 0.529^{\S} \\
\text { b versus c: } 0.001^{\S}\end{array}$ \\
\hline Mapping by side & & & & a versus b: $0.042 *$ \\
\hline None & $2(2.6 \%)$ & $6(15.8 \%)$ & 0 & a versus c: $0.003^{*}$ \\
\hline Unilateral & $30(39.0 \%)$ & $13(34.2 \%)$ & $7(14.6 \%)$ & $\mathrm{b}$ versus $\mathrm{c}: 0.0001^{*}$ \\
\hline Bilateral & $45(58.4 \%)$ & $19(50.0 \%)$ & $41(85.4 \%)$ & \\
\hline Patients with positive LN, $N(\%)$ & $18(23.4 \%)$ & $5(13.2 \%)$ & $8(16.7 \%)$ & $\begin{array}{l}\text { a versus b: } 0.149^{*} \\
\text { a versus c: } 0.253^{*} \\
\text { b versus c: } 0.445^{*}\end{array}$ \\
\hline Positive SLN, $N(\%)$ & $31(15.0 \%)$ & $8(12.9 \%)$ & $12(10.3 \%)$ & $\begin{array}{l}\text { a versus b: } 0.425^{*} \\
\text { a versus c: } 0.264^{*} \\
\text { b versus c: } 0.497^{*}\end{array}$ \\
\hline Type of metastasis, $N(\%)$ & & & & a versus b: $0.744^{*}$ \\
\hline Macrometastasis & $25(80.6)$ & $6(75.0)$ & $6(50.0)$ & a versus c: $0.101 *$ \\
\hline Micrometastasis & $4(12.9)$ & $2(25.0)$ & $5(41.7)$ & b versus c: $0.780^{*}$ \\
\hline ITC & $2(6.5)$ & 0 & $1(8.3)$ & \\
\hline
\end{tabular}

$L N$ lymph node, $S L N$ sentinel lymph node, ${ }^{99 m} T c$ technetium-99 radiocolloid, $I C G$ indocyanine green, $M I S$ minimally invasive surgery, $S H$ simple hysterectomy, BSO, PLND pelvic lymphadenectomy, PALND para-aortic lymphadenectomy, $R H$ radical hysterectomy, ITC isolated tumor cell

* Fisher's exact test

$\S$ Rank sum test

TABLE 3 SLN mapping according to tumor $(N=163)$

\begin{tabular}{|c|c|c|c|c|}
\hline \multirow[t]{2}{*}{ Characteristic } & \multirow{2}{*}{$\begin{array}{l}\text { Cervical }(N=45) \\
N(\%)\end{array}$} & \multirow{2}{*}{$\begin{array}{l}\text { Endometrial, }(n=118) \\
N(\%)\end{array}$} & \multicolumn{2}{|l|}{$p$ value } \\
\hline & & & Cervical & Endometrial \\
\hline SLN detection rate & & & NA & $0.004 *$ \\
\hline${ }^{99 m}$ Tc with blue due & $28 / 28(100 \%)$ & $42 / 44(95 \%)$ & & \\
\hline Blue dye alone & $8 / 8(100 \%)$ & $24 / 30(80 \%)$ & & \\
\hline ICG & 9/9 (100\%) & $40 / 40(100 \%)$ & & \\
\hline Bilateral mapping & & & $0.111 *$ & $0.004^{*}$ \\
\hline${ }^{99 m} \mathrm{Tc}$ with blue dye & $23 / 28(82 \%)$ & $22 / 44(50 \%)$ & & \\
\hline Blue dye alone & $5 / 8(63 \%)$ & $14 / 30(47 \%)$ & & \\
\hline
\end{tabular}


TABLE 3 continued

\begin{tabular}{llll}
\hline Characteristic & Cervical $(N=45)$ & Endometrial, $(n=118)$ & $p$ value \\
\cline { 2 - 3 } & $N(\%)$ & $N(\%)$ & Cervical \\
\hline ICG & $9 / 9(100 \%)$ & $32 / 40(80 \%)$ & Endometrial \\
\hline
\end{tabular}

SLN sentinel lymph node, $N A$ not available, ${ }^{99 m} T c$ technetium-99 radiocolloid, $I C G$ indocyanine green

* Fisher's exact test
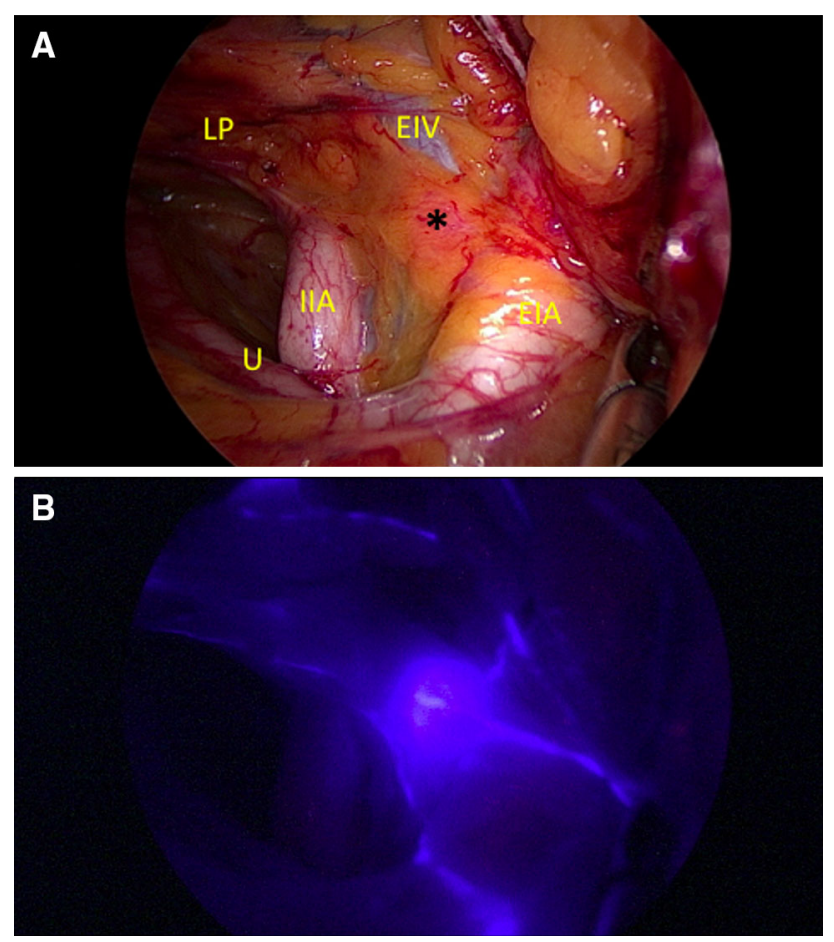

FIG. 2 Typical location of sentinel lymph node (SLN). a White light view of a SLN (asterisk) located in the ventral medial aspect of the external iliac artery (EIA). b The appearance of the SLN with the ICG real-time florescence Storz SPIES HD for laparoscopic platform. EIA external iliac artery, IIA internal iliac artery, $E I V$ external iliac vein, $U$ ureter, $L P$ lateral parametrium

$50 \%$ of ${ }^{99 \mathrm{~m}} \mathrm{Tc}$ with blue dye and blue dye alone, respectively). These differences are particularly evident and statistically significant in the case of endometrial cancer. The higher number of bilateral mappings - if confirmed in prospective trials - may consequently reduce the overall number of complete lymphadenectomies, thereby reducing the duration and additional costs of surgical treatment.

Although it was not the main aim of this study, our experience with ICG resulted in an improvement in the workflow because the SLN mapping procedure can be started directly in the operating room. This approach saves time and can avoid the discomfort for patients that results from the preoperative injection of radiocolloid in the nuclear medicine department. Furthermore, the use of ICG seems to be useful during the surgical procedure after SLN mapping, allowing the surgeon to complete the procedure without staining the operative field, as occasionally occurred with blue dye (Fig. 2). This is particularly useful in the case of obese patients, when bleeding covers the retroperitoneal fat and obscures the SLN, and the blue dye extensively stains the operative field. Moreover, the parametrial/paracervical region can be inspected easily using fluorescent dye without impairing parametrial dissection.

The retrospective European data available regarding the use of ICG for SLN mapping are limited. In 2015 Plante et al. and Imboden et al. presented their pilot experiences with ICG in uterine and cervical cancer. ${ }^{23,25}$ Among the 379 published cases mapped with ICG, the overall DR and the rate of bilateral mapping was better compared to the available literature using ${ }^{99 \mathrm{~m}} \mathrm{Tc}$ radiocolloid and blue dye. By comparing ${ }^{99 \mathrm{~m}} \mathrm{Tc}$, blue dye, and $\mathrm{ICG}$, How et al. recently supported the results of the Memorial Sloan Kettering group suggesting that blue dye may be omitted for SLN mapping in endometrial cancer. ${ }^{26}$

Our results are similar to those recently published demonstrating that SLN mapping with ICG fluorescence seems to improve SLN mapping and bilateral mapping better than with ${ }^{99 \mathrm{~m}} \mathrm{Tc}$ with blue dye or blue dye alone for both cervical and endometrial cancers.

Even if the data were recorded consecutively and if there was no difference in the baseline characteristics within the three groups, our study has some limitations because of the small sample size and because of its nonrandomized nature. No patients with negative SLNs with positive non-SLNs for metastases were found; however, the value of a false-negative rate of 0 could be underestimated because in our study, a systematic pelvic and aortic lymphadenectomy was performed only in the case of failed mapping and in the case of positive PET/CT in the pelvic and/or aortic chains of endometrial cancer patients. However, this approach was based on the results described in the current literature, which shows an estimated risk of almost 2-3\% of isolated aortic metastases in preoperative endometrial stage I disease. ${ }^{27,28}$ Furthermore, the learning curve associated with the use of a new technique probably 
decreases the bilateral DR seen at the beginning of our experience using ${ }^{99 \mathrm{~m}} \mathrm{Tc}$ and blue dye. ${ }^{13}$

\section{CONCLUSIONS}

On the basis of our preliminary data, cervical injection of ICG seems to be a reliable technique for SLN mapping in patients with early stage cervical cancer and preoperative stage I endometrial cancer. The laparoscopic fluorescent device and the Vitom II exoscope in an open surgical approach provides good results and may be widely applicable in many centers where the robotic platform is not available. SLN mapping with a well-defined algorithm represents an acceptable surgical staging approach.

The concept of tumor staging is a process that is continuously changing and is destined to evolve and improve, thereby allowing us to learn more about how to provide better care for women with early stage cervical and endometrial cancer.

ACKNOWLEDGMENT We thank Patricia Ann Sawchuk for her friendship and contribution to the article's final revision.

DISCLOSURE The authors declare no conflict of interest.

\section{REFERENCES}

1. Van der Zee AG, Oonk MH, De Hullu JA, et al. Sentinel node dissection is safe in the treatment of early-stage vulvar cancer. $J$ Clin Oncol. 2008;26:884-9.

2. Oonk MH, van Hemel BM, Hollema H, et al. Size of sentinelnode metastasis and chances of non-sentinel-node involvement and survival in early stage vulvar cancer: results from GROINSS$\mathrm{V}$, a multicentre observational study. Lancet Oncol. 2010;11:646-52.

3. Khoury-Collado F, Murray MP, Hensley ML, et al. Sentinel lymph node mapping for endometrial cancer improves the detection of metastatic disease to regional lymph nodes. Gynecol Oncol. 2011;122:251-4.

4. Ballester M, Naoura I, Chéreau E, et al. Sentinel node biopsy upstages patients with presumed low- and intermediate-risk endometrial cancer: results of a multicenter study. Ann Surg Oncol. 2013;20:407-12.

5. Cormier B, Diaz JP, Shih K, et al. Establishing a sentinel lymph node mapping algorithm for the treatment of early cervical cancer. Gynecol Oncol. 2011;122:275-80.

6. Burke TW, Levenback C, Tornos C, Morris M, Wharton JT, Gershenson DM. Intraabdominal lymphatic mapping todirect pelvic and aortic lymphadenectomy in women with high risk endometrial cancer: results of a pilot study. Gynecol Oncol. 1996;62:169-73.

7. van de Lande J, Torrenga B, Raijmakers PG, et al. Sentinel lymph node detection in early stage uterine cervix carcinoma: a systematic review. Gynecol Oncol. 2007;106:604-13.

8. Ballester M, Dubernard G, Rouzier R, Barranger E, Darai E. Use of the sentinel node procedure to stage endometrial cancer. Ann Surg Oncol. 2008;15:1523-9.
9. Daraï E, Dubernard G, Bats AS, et al. Sentinel node biopsy for the management of early stage endometrial cancer: long-term results of the SENTI-ENDO study. Gynecol Oncol. 2015;136:549.

10. Jewell EL, Huang JJ, Abu-Rustum NR, et al. Detection of sentinel lymph nodes in minimally invasive surgery using indocyanine green and near-infrared fluorescence imaging for uterine and cervical malignancies. Gynecol Oncol. 2014;288(133):274-7.

11. Xiong L, Gazyakan E, Yang W, Engel H, Hunerberin M, Kneser U. Indocyanine green fluorescence-guided sentinel node biopsy: a meta-analysis on detection rate and diagnostic performance. Eur J Surg Oncol. 2014;40:843-9.

12. Schaafsma BE, Verbeek FP, Elzevier HW, et al. Optimization of sentinel lymph node mapping in bladder cancer using near-infrared fluorescence imaging. J Surg Oncol. 2014;110:845-50.

13. Buda A, Elisei F, Arosio M, et al. Integration of hybrid singlephoton emission computed tomography/computed tomography in the preoperative assessment of sentinel node in patients with cervical and endometrial cancer: our experience and literature review. Int J Gynecol Cancer. 2012;22:830-5.

14. Horn LC, Einenkel J, Hockel M, et al. Recommendations for the handling and oncologic pathology report of lymph node specimens submitted for evaluation of metastatic disease in gynecologic malignancies. Pathologe. 2005;26:266-72.

15. Granzow JW, Soderberg JM, Kaji AH, et al. Review of current surgical treatments for lymphedema. Ann Surg Oncol. 2014;21:1195-201.

16. Raimond E, Ballester M, Hudry D, et al. Impact of sentinel lymph node biopsy on the therapeutic management of early-stage endometrial cancer: results of a retrospective multicenter study. Gynecol Oncol. 2014;133:506-11.

17. Leitao MM, Khoury-Collado F, Gardner G, et al. Impact of incorporating an algorithm that utilizes sentinel lymph node mapping during minimally invasive procedures on the detection of stage IIIC endometrial cancer. Gynecol Oncol. 2013;129:3841.

18. NCCN Clinical Practice Guidelines in Oncology (NCCN Guidelines). Version 2.2015.

19. Koh WJ, Greer BE, Abu-Rustum NR, et al. Cervical cancer, version 2.2015. J Natl Compr Canc Netw. 2015;13:395-404.

20. Barlin JN, Khoury-Collado F, Kim CH, et al. The importance of applying a sentinel lymph node mapping algorithm in endometrial cancer staging: beyond removal of blue nodes. Gynecol Oncol. 2012;125:531-5.

21. Cibula D, Abu-Rustum NR, Dusek L, et al. Prognostic significance of low volume sentinel lymph node disease in early-stage cervical cancer. Gynecol Oncol. 2012;124:496-501.

22. Sinno AK, Fader AN, Roche KL, Giuntoli RL 2nd, Tanner EJ. A comparison of colorimetric versus fluorometric sentinel lymph node mapping during robotic surgery for endometrial cancer. Gynecol Oncol. 2014;134:281-6.

23. Plante M, Touhami O, Trinh XB, et al. Sentinel node mapping with indocyanine green and endoscopic near-infrared fluorescence imaging in endometrial cancer. A pilot study and review of the literature. Gynecol Oncol. 2015;137:443-7.

24. Handgraaf HJ, Verbeek FP, Tummers QR, et al. Real-time nearinfrared fluorescence guided surgery in gynecologic oncology: a review of the current state of the art. Gynecol Oncol. 2014;135:606-13.

25. Imboden S, Papadia A, Nauwerk M, et al. A comparison of radiocolloid and indocyanine green fluorescence imaging, sentinel lymph node mapping in patients with cervical cancer undergoing laparoscopic surgery. Ann Surg Oncol. 2015. doi:10. 1245/s10434-015-4701-2. 
26. How J, Gotlieb WH, Press JZ, et al. Comparing indocyanine green, technetium, and blue dye for sentinel lymph node mapping in endometrial cancer. Gynecol Oncol. 2015. doi:10.1016/j. ygyno.2015.04.004.

27. Abu-Rustum NR, Gomez JD, Alektiar KM, et al. The incidence of isolated paraaortic nodal metastasis in surgically staged endometrial cancer patients with negative pelvic lymph nodes. Gynecol Oncol. 2009;115:236-8.

28. Chiang $\mathrm{AJ}, \mathrm{Yu} \mathrm{KJ}$, Chao $\mathrm{KC}$, et al. The incidence of isolated para-aortic nodal metastases in completely staged endometrial cancer patients. Gynecol Oncol. 2011;121:122-5. 